\title{
Parametric study for aircraft impact
}

\author{
L. E. Laczák ${ }^{1} \&$ G. Károlyi ${ }^{2}$ \\ ${ }^{I}$ Department of Structural Engineering, \\ Budapest University of Technology and Economics, Hungary \\ ${ }^{2}$ Institute of Nuclear Techniques, \\ Budapest University of Technology and Economics, Hungary
}

\begin{abstract}
The Riera approach is the most commonly used method for modelling the global effects of a deformable missile on a rigid engineering structure. The Riera approach assumes the normal impact of a rigid perfectly plastic missile that only crushes at the cross-section adjacent to the target. It also neglects the effects of target deformation. Our research focuses on analysing the limits of the applicability of the Riera model by comparing its results to that of a finite element (FE) model. Different initial parameters of impact, missile and target are considered over a wide range of values.

Keywords: aircraft impact, global effect, Riera-approach.
\end{abstract}

\section{Introduction}

\subsection{Riera model}

If the target structure is rigid enough then the effects of its deformations on the reaction force during an aircraft impact can be neglected and the impact can be modelled as a time dependent loading function acting on the target. This means that in order to analysis the effects of an impact on a structure firstly the impact force has to be determined and secondly it has to be applied on the target structure. According to Riera [1] the loading function obtained this way depends only on the initial parameters of the aircraft. Assumptions of the Riera-approach (fig. 1):

- $\quad$ the target is perfectly rigid (target velocity $v_{t}=0$ );

- the impact is in normal direction; 
- material of the aircraft is rigid-perfectly plastic (no elastic deformations or shockwaves occur);

- the aircraft consists of an uncrushed and the actually crushing part (of mass $m$ and $d m$, respectively);

- the aircraft crushes only at the cross-section adjacent to the target having the length $d x$ and mass $d m$;

- $\quad$ during the impact $d x$ breaks down and then its velocity reduces to 0 .

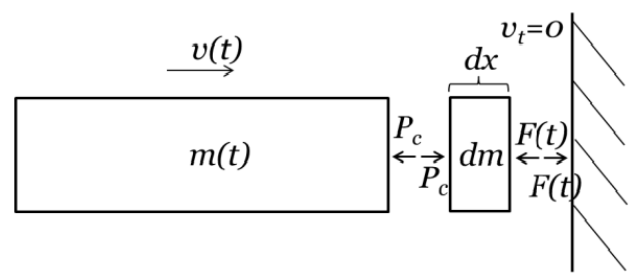

Figure 1: Riera model for aircraft impact into a rigid target (adapted form [1]).

If $\mu(x)$ and $P_{c}(x)$ are the distributed mass and crushing force along the length of the aircraft ( $x$ is the distance of the actually crushing cross-section measured from the original position of the nose), then the $F(t)$ reaction force acting on the target is [1]:

$$
F(t)=P_{c}(x)+\mu(x) \cdot(\dot{x})^{2} .
$$

Equation (1) shows that the impact force at time $t$ depends only on the velocity of crushing and on the properties of the actually crushing cross-section of the aircraft.

\subsection{Finite element (FE) model}

Parallel to calculations carried out by the Riera model, ANSYS LS-DYNA analyses are also executed. LS-DYNA uses explicit dynamic analysis for solution of high velocity, large deformation, non-linear contact problems. In our models 3D SOLID164 elements and bilinear material model are used for the aircraft (fig. 2) and perfectly rigid material model for the target. In the FE model elements start to yield at limit stress $f_{c}$ and their failure occurs when the strain reaches an ultimate value $\varepsilon_{u}$.

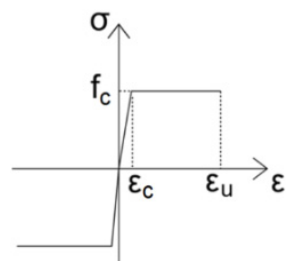

Figure 2: $\quad$ Material model of aircraft. 
Input values for the FE model, namely the density $\rho$ and limit stress $f_{c}$ of the aircraft material can be directly calculated if $P_{c}$ and $\mu$ are known, while $\varepsilon_{u}$ only appears in the FE model, so its value is determined from our previous tests $[2,3]$.

\section{Comparison of the Riera and the FE models}

In order to verify the applicability of the Riera and the FE models their results are compared to those of a real-size experiment executed by Sugano et al. [4]. During this test an F4-Phantom military aircraft was accelerated to $215 \mathrm{~m} / \mathrm{s}$ by the aid of a special rocket sled and crashed into a rigid concrete block that had the size of $7 \times 7 \times 3.66 \mathrm{~m}$. High-speed cameras, accelerometers, displacement and velocity gauges were used to measure the accelerations, velocities, displacements during the impact. In order to provide frictionless motion for the target, air bearings were installed between the concrete block and its platform. It was observed that after the impact only the concrete cover was damaged at the impact zone, so little energy went into structural deformations of the target, therefore the impact force acting on the target could be simply calculated from the accelerations. From the impact force and the mass distribution of the aircraft the crushing force could be obtained by backward Riera method calculation (eqn. (1)).

In order to test the applicability of different models, an aircraft is built in LSDYNA that has the input parameters (geometry, mass and crushing force distribution) close to those of the F4-Phantom used by Sugano (fig. 3). Calculations are made by the Riera model and the FE model, for which the input parameters are the same. In fig. 4 and fig. 5 input data (distributed mass and crushing force) are represented and are compared to real aircraft data of the Phantom fighter used by Sugano.
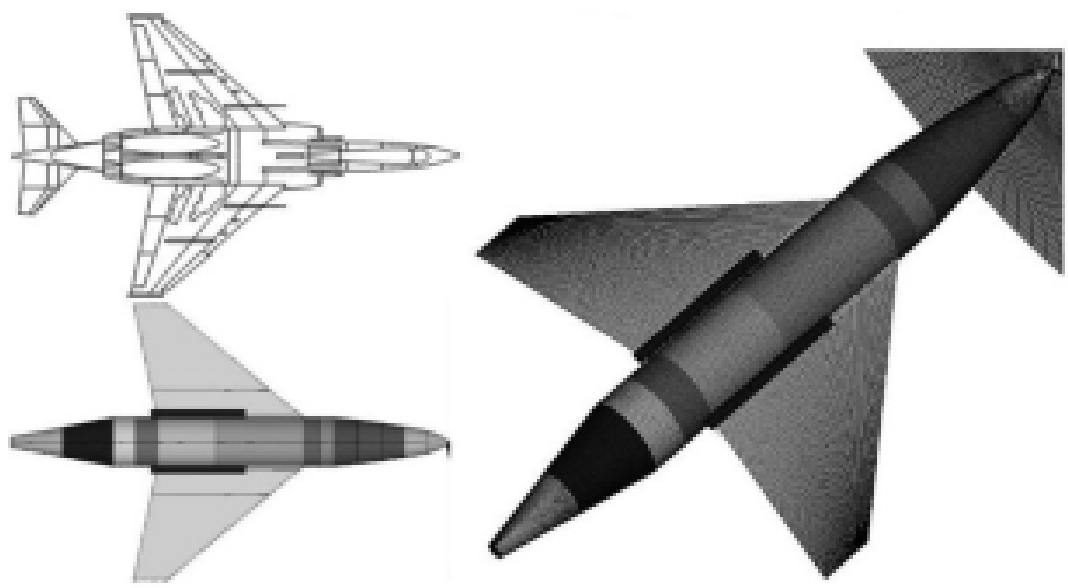

Figure 3: $\quad$ Top view of a real F4-Phantom [5] and pictures from FE model. 


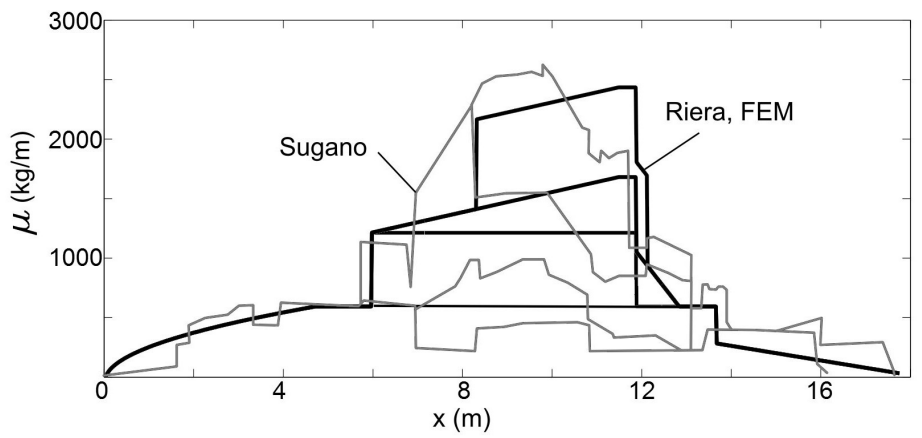

Figure 4: Distributed mass along the length of aircraft (mass of different parts are also represented).

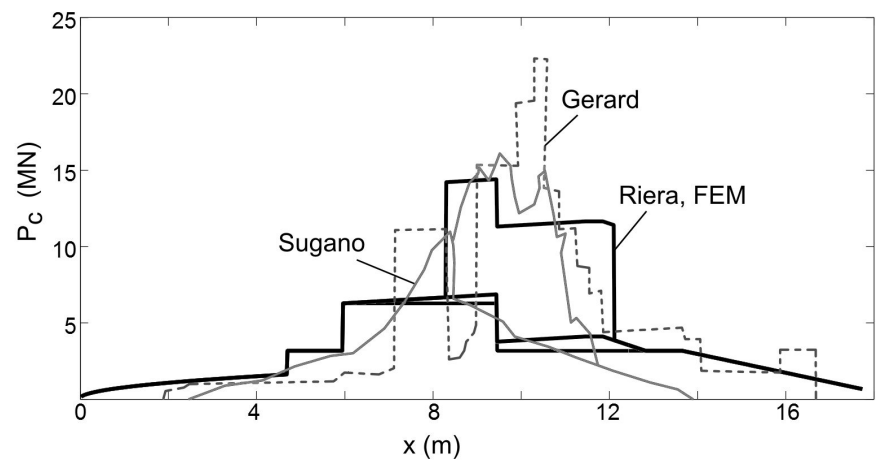

Figure 5: Crushing force along the length of aircraft.

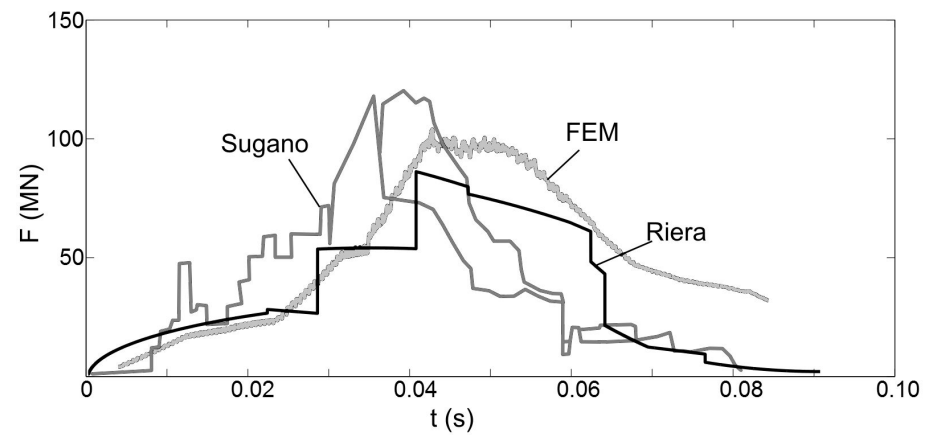

Figure 6: Reaction force - time curve measured by Sugano [4] and calculated by Riera-model and FE model.

In fig. 6 as a result of our calculations, the reaction force-time diagram can be seen. If Riera model, FE model and real impact are compared, it can be stated that results obtained from similar input data are in good agreement. 


\section{Effect of aircraft nose and tail}

Based on our previous calculations $[2,3]$ it can be stated that the beginning and final part of the impact shows significant differences in the Riera and the FE model therefore the effect of nose and tail mass and rigidity has to be analysed. In order to analyse effect of nose and tail, four different models are used, in which wings and engines are not modelled for simplicity. Our models are:

- model_0: hollow cylindrical missile that has constant unit mass and crushing force along the whole length;

- model_1: cylindrical fuselage with nose and tail, constant density and limit stress;

- model_2: cylindrical fuselage with nose and tail, limit stress of nose and tail is half of the limit stress of the fuselage;

- model 3: cylindrical fuselage with nose and tail, limit stress and density of nose and tail are half of the values used for the fuselage.

The approximate fuselage, nose and tail geometry and average parameter values of the F4-Phantom introduced in the previous section are used for our calculations, and also the same impact velocity $(215 \mathrm{~m} / \mathrm{s})$ is applied. The model geometry can be seen in fig. 7 and input parameters of the models are listed in Table 1.
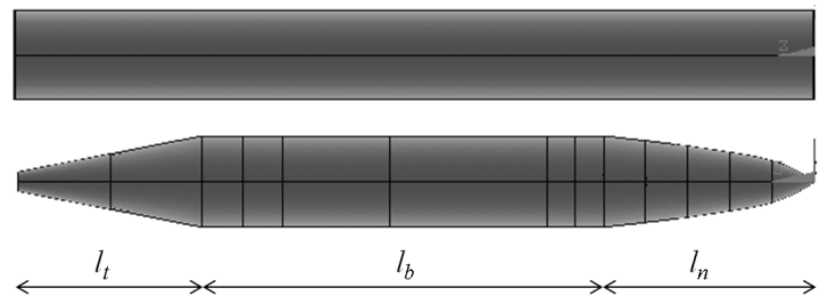

Figure 7: Top view of model_0 (top) and model_1-3 (bottom) (black lines are guidelines used during the modelling).

Table 1: Input parameters for our models.

\begin{tabular}{|c|c|c|c|c|c|c|c|c|}
\hline \multicolumn{9}{|c|}{$\begin{array}{l}l_{n}: \text { length of nose, } l_{f}: \text { length of cylindrical fuselage, } l_{t}: \text { length of tail, } r: \text { radius of } \\
\text { fuselage, } \rho: \text { density of fuselage, } \rho_{n}=\rho_{t}: \text { density of nose and tail, } f_{c}: \text { limit stress } \\
\text { of fuselage, } f_{c n}=f_{c t}: \text { limit stress of nose and tail }\end{array}$} \\
\hline sign & $\begin{array}{l}l_{n} \\
(m)\end{array}$ & $\begin{array}{l}l_{f} \\
(m)\end{array}$ & $\begin{array}{c}l_{t} \\
(m)\end{array}$ & $\begin{array}{c}r \\
(m)\end{array}$ & $\begin{array}{c}\rho \\
\left(\mathrm{kg} / \mathrm{m}^{3}\right)\end{array}$ & $\begin{array}{c}\rho_{n}=\rho_{t} \\
\left(\mathrm{~kg} / \mathrm{m}^{3}\right)\end{array}$ & $\begin{array}{c}f_{c} \\
(M N / m)\end{array}$ & $\begin{array}{l}f_{c n}=f_{c t} \\
(M N / m)\end{array}$ \\
\hline model 0 & 0 & 17.7 & 0 & 1.0 & 5912 & 5912 & 29.8 & 290.8 \\
\hline model_1 & 4.7 & 8.9 & 4.1 & 1.0 & 5912 & 5912 & 29.8 & 29.8 \\
\hline model 2 & 4.7 & 8.9 & 4.1 & 1.0 & 5912 & 5912 & 29.8 & 14.9 \\
\hline model 3 & 4.7 & 8.9 & 4.1 & 1.0 & 5912 & 2956 & 29.8 & 14.9 \\
\hline
\end{tabular}


Reaction force - time diagrams of model_0-3 are represented in fig. 8 and fig. 9, Results of the Riera model are drawn by solid lines, while dashed lines show the moving averages of the output values of the FE model. Fig. 8 shows that if nose and tail geometry is taken into account then the Riera and the FE model results are closer to each other.

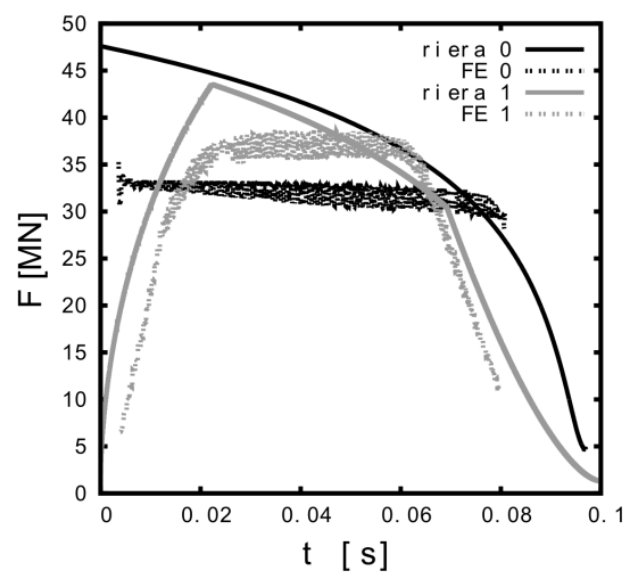

Figure 8: Reaction force - time curve of model_0 and model_1, calculated by the Riera and the FE models.

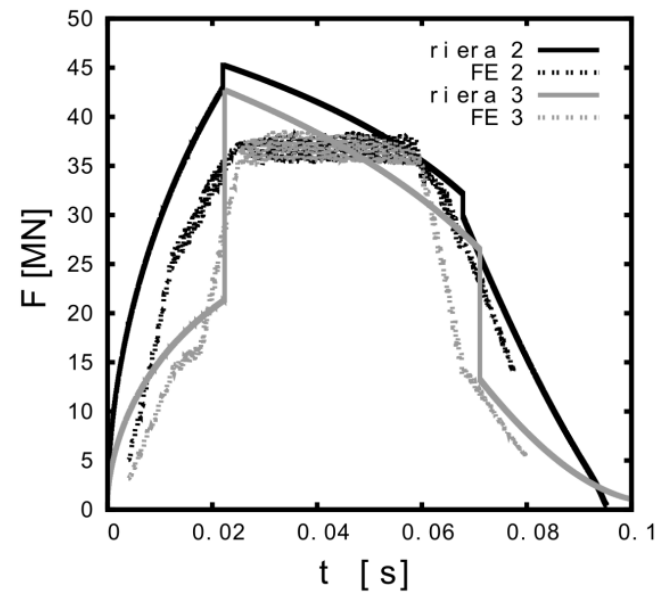

Figure 9: Reaction force - time curve of model_2 and model_3, calculated by the Riera and the FE models.

If model_2 and model_3 results are compared to model_1 it is visible that density has more significant effect on the reaction force in the Riera and the FE model results, than limit stress. This result is in agreement with the fact that in 
eqn. (1). the second term is dominant and the reaction force (at $215 \mathrm{~m} / \mathrm{s}$ ) can be 8-10 times higher than the crushing force. Riera and FE model results for model_2 and model_3 harmonise quite well, difference between peak values is

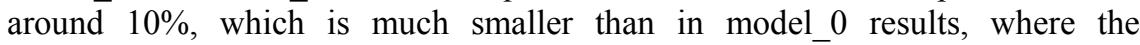
difference of maximum values is $30 \%$.

\section{Conclusion}

This paper mainly focused on testing the applicability of the Riera-model and a finite element model for aircraft impact. After harmonising the input parameters of the models with a real-size impact test's parameters, the reaction-time functions were calculated. The results of our calculations agreed quite well with the results measured during the real test.

In the second part of our study, the effect of nose and tail was tested and it could be stated that Riera and FE model results and their differences highly depend on the properties of the nose and tail of the aircraft.

\section{Acknowledgement}

This project is supported by the Hungarian Scientific Research Fund (OTKA K100894).

\section{References}

[1] Riera, J.D., On the stress analysis of structures subjected to aircraft impact forces, Nuclear Engineering and Design, 8, pp. 415-426, 1968.

[2] Laczák, L.E., Aircraft impact into rigid structure, Proc. of the Second Conference of Junior Researchers in Civil Engineering, eds. J. Józsa, R. Németh, T. Lovas, Budapest, pp. 105-110, 2013.

[3] Laczák, L.E., Károlyi, Gy., Aircraft Impact into reinforced concrete structure, Book of Abstracts, Nonlinear Dynamics in Engineering: Modelling, Analysis and Applications, eds. M. Wiercigroch, E. Pavlovskaia, J. Ing, Y. Liu, A. Postnikov, Aberdeen, pp. 36, 2013.

[4] Sugano, T., Tsubota, H., Kasai, Y., Koshika, N., Orui, S., Riesemann, W.A., Bickel, D.C., Parks, M.B.: Full-scale aircraft impact test for evaluation of impact force, Nuclear Engineering and Design, 140, pp. 373-385, 1993.

[5] Views of F-4 Phantom: http://en.wikipedia.org/wiki/File:McDONNELL _DOUGLAS_F-4_PHANTOM_II.png 